\title{
3D Real Inversion Recovery MR Imaging for the Visualization of Endolymphatic Hydrops
}

W e read with interest the article by Baráth et $\mathrm{al}^{1}$ describing the reliability of MR imaging performed 4 hours after intravenous administration of double-dose gadolinium-based contrast agent for the visualization of endolymphatic hydrops in Menière disease. Although we agree with the principal findings of the study, we would like to clarify an important technical point to further increase the practical value of the article. The authors stated that they used a $3 \mathrm{D}$ real inversion recovery (3D real IR) sequence using our previously reported method. ${ }^{2}$ Real inversion recovery is the method that allows the negative magnetizations before crossing the null point are correctly represented as negative values by the use of phase-sensitive reconstruction. ${ }^{3}$ In the MR images shown, endolymphatic fluid shows near zero signal intensity, similar to that of surrounding bone, instead of a negative signal-intensity value as expected on 3D real IR. 3D real IR was intended to separately visualize endolymph, perilymph, and bone on a single kind of image. ${ }^{2} \mathrm{MR}$ images shown in the article are more like 3D FLAIR than 3D real IR. Clarifi-

http://dx.doi.org/10.3174/ajnr.A4126 cation of this technical point might be very important for the readers to reproduce this important study.

\section{REFERENCES}

1. Baráth K, Schuknecht B, Naldi AM, et al. Detection and grading of endolymphatic hydrops in Menière disease using MR imaging. AJNR Am J Neuroradiol 2014;35:1387-92

2. Naganawa S, Ishihara S, Iwano S, et al. Three-dimensional (3D) visualization of endolymphatic hydrops after intratympanic injection of Gd-DTPA: optimization of a 3D-real inversion-recovery turbo spin-echo (TSE) sequence and application of a 32-channel head coil at 3T. J Magn Reson Imaging 2010;31:210-14

3. Park HW, Cho MH, Cho ZH. Real-value representation in inversion-recovery NMR imaging by use of a phase-correction method. Magn Reson Med 1986;3:15-23

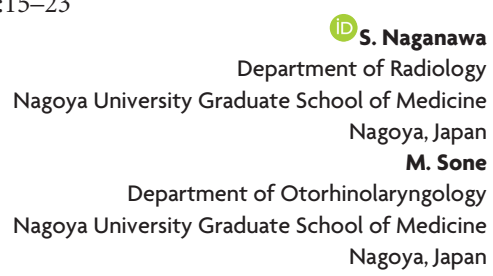

